01

\title{
Гелиевое послесвечение без метастабильных частиц
}

\author{
() В.А. Иванов, Ю.Э. Скобло \\ Санкт-Петербургский государственный университет, \\ 198504 Санкт-Петербург, Россия \\ e-mail: v.a.ivanov@spbu.ru, yuri_skoblo@mail.ru \\ Поступила в редакцию 08.05.2019 г. \\ В окончательной редакции 25.07.2019 г. \\ Принята к публикации 12.08.2019 г.
}

Исследовано послесвечение импульсного барьерного разряда в гелии с малой примесью неона $\left(10^{-2} \%\right)$, создающего плазму с малой плотностью метастабильных частиц. Ранняя стадия послесвечения такого разряда свободна от процессов с участием метастабильных частиц и имеет чисто рекомбинационную природу. Характеристики послесвечения интерпретируются на основании модели с учетом колебательной кинетики и диссоциативной рекомбинации молекулярных ионов $\mathrm{He}_{2}^{+}$. Сопоставление экспериментальных данных и модельных решений для потоков ударно-радиационной рекомбинации ионов $\mathrm{He}^{+}$с электронами и диссоциативной рекомбинации приводит к выводу в пользу последнего процесса как источника образования возбужденных атомов.

Ключевые слова: послесвечение разряда в гелии, метастабильные атомы, диссоциативная рекомбинация.

DOI: $10.21883 /$ OS.2019.12.48681.156-19

\section{Введение}

Процессы электрон-ионной рекомбинации играют важную роль в физике плазмы и астрофизике, в формировании свойств газоразрядной плазмы и особенно активных сред плазменных лазеров. Основным каналом деионизации плазмы, содержащей молекулярные ионы, является их диссоциативная рекомбинация с электронами. Исследованию этого процесса, начиная с 1949 г. [1], посвящено множество работ. Так, число цитированных исследований в обзоре [2] приближается к четыремстам. Механизм диссоциативной рекомбинации является необходимым звеном моделей звездных и планетарных атмосфер [3], развитию теоретических подходов к его описанию уделяется большое внимание [4].

Процессы в газоразрядной гелиевой плазме, определяющие скорость ее деионизации, принципиально отличаются от процессов в плазме других инертных газов. Вопервых, в гелиевой плазме концентрации атомов в метастабильных состояниях $\mathrm{He}\left(2^{3} S_{1}, 2^{1} S_{0}\right)$ (в дальнейшем $\left.\mathrm{He}_{m}\right)$, как правило, на порядок величины превышают плотность электронов [е] [5]. Как стало известно уже из первых экспериментов [6], в стадии распада плазмы столкновения этих атомов:

$$
\mathrm{He}_{m}+\mathrm{He}_{m} \rightarrow \mathrm{He}^{+}+\mathrm{He}+e \rightarrow \mathrm{He}_{2}^{+}+e,
$$

создают мощный поток ионизации, маскирующий рекомбинационные процессы. К тому же энергия образующихся в реакции (1) „быстрых“ электронов достаточно велика для ступенчатой ионизации и возбуждения атомов гелия. Во-вторых, вследствие специфики потенциалов взаимодействия возбужденных атомов гелия Не* с атомами в основном состоянии отсутствует благоприятное для диссоциативной рекомбинации (DR)

$$
\mathrm{He}_{2}^{+}(v)+e \stackrel{\alpha_{\mathrm{DR}(j)}}{\longrightarrow} \mathrm{He}^{*}(j)+\mathrm{He}
$$

$\left(\alpha_{\mathrm{DR}}-\right.$ коэффициент рекомбинации $)$ пересечение термов $\mathrm{He}^{*}+\mathrm{He}$ и $\mathrm{He}_{2}^{+}$вблизи наиболее населенного основного колебательного уровня $v=0$ молекулярного иона [7]. Наконец, в гелиевой плазме образуются метастабильные молекулы $\mathrm{He}_{2}^{*}\left(2 s^{3} \Sigma_{u}^{+}\right)$, которые также участвуют в процессах типа (1). Все это сильно затрудняет исследование механизма DR (2), детально изученного в других системах, и понимание его роли в распадающейся слабоионизованной гелиевой плазме. Как неоднократно отмечалось в литературе $[8,9]$, обилие исследований в данном случае не способствовало прояснению ситуации. Коэффициенты DR при температуре электронов $T_{e}=300 \mathrm{~K}$ по данным различных авторов отличаются в десятки раз: от $\alpha_{\mathrm{DR}} \approx 10^{-8} \mathrm{~cm}^{3} / \mathrm{s}$ в ранних работах $[1,10,11]$ до $\leq 5 \cdot 10^{-10} \mathrm{~cm}^{3} / \mathrm{s}$ в [5]. В экспериментах [12], выполненных с помощью техники Test Storage Ring, получено $\alpha_{\mathrm{DR}} \approx 3 \cdot 10^{-10} \mathrm{~cm}^{3} / \mathrm{s}$ для $\mathrm{He}_{2}^{+}(v=0)$ и $\alpha_{\mathrm{DR}} \geq 2 \cdot 10^{-7} \mathrm{~cm}^{3} / \mathrm{s}$ для ионов $\mathrm{He}_{2}^{+}(v \geq 3)$. Похожие результаты предлагает и теоретический анализ [9], различающий эффективность рекомбинации ионов в различных колебательных состояниях: величины масштаба $5 \cdot 10^{-11} \mathrm{~cm}^{3} / \mathrm{s}$ для ионов $\operatorname{He}_{2}^{+}(v=0)$ и значительно более высокие скорости DR в случае колебательновозбужденных ионов $\mathrm{He}_{2}^{+}(v \geq 3,4)$, характерные для молекулярных ионов других инертных газов.

Не менее противоречивы и результаты спектроскопического анализа гелиевого послесвечения. До настоящего времени не сформировалась единая точка зрения даже по вопросу о механизмах образования возбужденных атомов в распадающейся гелиевой плазме. Выводы первых исследований, связывавших послесвечение 
c DR [13,14], впоследствии были пересмотрены [15] в пользу гипотезы о доминирующей роли ударнорадиационной рекомбинации ионов $\mathrm{He}^{+}$.

В настоящей работе представлены результаты спектроскопического исследования гелиевого послесвечения, свободного от процессов с участием метастабильных частиц. Показано, что адекватное описание послесвечения достигается в рамках модели диссоциативной рекомбинации как источника возбужденных атомов.

\section{Эксперимент}

В качестве объекта исследования использовалась распадающаяся гелиевая плазма с небольшой $\left(\approx 10^{-2 \%}\right)$ добавкой неона, инициированная диэлектрическим барьерным разрядом (DBD) в цилиндрической стеклянной трубке с электродами (полоски медной фольги) на ее внешних боковых поверхностях (рис. 1) при давлениях десятки Torr [16] и частотах десятки-сотни Hz. B [16] показано, что по сравнению как с импульсным разрядом между электродами внутри трубки, так и внешними электродами на вертикальных отростках трубки (при этом электроды DBD снимались), как показано на рис. 1, DBD такой конфигурации создает на оси плазму с плотностью метастабильных атомов $\left[\mathrm{He}\left(2^{1} S_{0}\right)\right]$ почти в 3 раза, $\left[\operatorname{He}\left(2^{3} S_{1}\right)\right]$ - в 4 раза меньшей при той же плотности электронов. При этом плотность метастабильных молекул гелия также оказывается пониженной. Это радикально меняло картину раннего послесвечения в атомном и молекулярном спектрах. В настоящей работе представлены некоторые результаты анализа и моделирования послесвечения такого разряда.

Плотность электронов в начальной стадии послесвечения оценивали по скорости спада $\left[\mathrm{He}\left(2{ }^{1} S_{0}\right)\right](t)$, обусловленного процессом:

$$
\mathrm{He}\left(2^{1} S_{0}\right)+e \stackrel{k_{e}}{\longrightarrow} \mathrm{He}\left(2^{3} S_{1}\right)+e,
$$

который является в данных условиях основным каналом их разрушения. Константа $k_{e}$ скорости процесса (3) известна [17] $k_{e}=3.5 \cdot 10^{-7} \mathrm{~cm}^{3} / \mathrm{s}$ и не зависит от температуры электронов [18], которая может меняться в ранней стадии послесвечения. Интенсивности линий Не и $\mathrm{Ne}$ на выходе монохроматора с разрешением $0.05 \mathrm{~nm}$ регистрировались 2048-канальным счетчиком фотонов с необходимым временным разрешением. Помимо интенсивностей спектральных линий и молекулярных полос $\mathrm{He}_{2}^{*}$, в работе измерялось поглощение на линиях $501.57 \mathrm{~nm}\left(2^{1} S \leftarrow 3^{1} P\right)$ и $388.9 \mathrm{~nm}\left(2^{3} S \leftarrow 3^{3} P\right)$ и вычислялись абсолютные величины плотностей $\left[\mathrm{He}\left(2^{1} S_{0}\right)\right]$ и $\left[\operatorname{He}\left(2^{3} S_{1}\right)\right]$.

\section{Результаты и обсуждение}

На рис. 2 показан временной ход наиболее ярких линий гелия $587.6 \mathrm{~nm}\left(2^{3} P \leftarrow 3^{3} D\right)$ и неона $585.2 \mathrm{~nm}$
Плотности атомов в метастабильных состояниях в начале послесвечения двух разрядов

\begin{tabular}{c|c|c}
\hline Разряд & $\begin{array}{c}{\left[\mathrm{He}\left(2^{1} S_{0}\right],\right.} \\
10^{11} \mathrm{~cm}^{-3}\end{array}$ & $\begin{array}{l}{\left[\mathrm{He}\left(2^{3} S_{1}\right],\right.} \\
10^{11} \mathrm{~cm}^{-3}\end{array}$ \\
\hline DBD & 0.45 & 1.3 \\
El & 1.2 & 5.2
\end{tabular}

$\left(1 s_{2} \leftarrow 2 p_{1}\right.$ по Пашену) в послесвечении обоих разрядов при давлении гелия 19 Torr. Параметры разрядов подобраны так, чтобы плотность электронов в начале послесвечения в обоих случаях была одинаковой $[\mathrm{e}] \approx 8 \cdot 10^{10} \mathrm{~cm}^{-3}$. Видно, что раннее гелиевое послесвечение с уменьшенной плотностью метастабильных частиц (таблица) имеет принципиально иной характер. Подчеркнем, что это не является следствием каких-либо постразрядных явлений, например, изменения плотности газа вследствие его нагрева, а обусловлено элементарными процессами в плазме, на что указывают сравнительно небольшие отличия в послесвечении неоновых линий.

Для интерпретации наблюдаемых различий мы об-

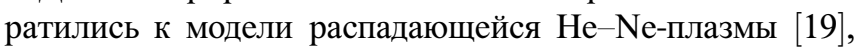
представив в ней $\mathrm{He}_{2}^{+}$как молекулярный ион, имеющий три состояния: $\operatorname{He}_{2}^{+}(v=0), \operatorname{He}_{2}^{+}(v=1)$ и $\operatorname{He}_{2}^{+}(v=2)$, населенности которых описываются соответствующими дифференциальными уравнениями.

Вследствие процессов типа (1) и механизма конверсии:

$$
\mathrm{He}^{+}+\mathrm{He}+\mathrm{He} \stackrel{k_{c}}{\longrightarrow} \mathrm{He}_{2}^{+}(v=2)+\mathrm{He}
$$

(константа скорости процесса $k_{c}=(6.2 \pm 0.5)$. $\cdot 10^{-32} \mathrm{~cm}^{3} / \mathrm{s}$ [5]) молекулярные ионы образуются в высоковозбужденных колебательных состояниях [5], поэтому соответствующий поток мы направили на верхний в модели колебательный уровень $v=2$, в то время как в рекомбинации с электронами участвуют ионы $\operatorname{He}_{2}^{+}(v=1)$, формируя атомный спектр, и $\mathrm{He}_{2}^{+}(v=0)$ как источник молекулярного спектра. Таким образом, в модели поток DR равен:

$$
F_{\mathrm{DR}}=\alpha_{\mathrm{DR}}\left[\mathrm{He}_{2}^{+}(v=1)\right][\mathrm{e}],
$$

где в качестве коэффициента рекомбинации колебательно-возбужденных ионов мы взяли в соответствии с [12] (см. выше) $\alpha_{\mathrm{DR}}=2 \cdot 10^{-7} \mathrm{~cm}^{3} / \mathrm{s}$. Поток (5) сравниваем c потоком ударно-радиационной рекомбинации (CRR) ионов $\mathrm{He}^{+}$:

$$
F_{\mathrm{CRR}}=\alpha_{\mathrm{CRR}}\left[\mathrm{He}^{+}\right][\mathrm{e}],
$$

в расчетах которого использована предложенная в [20] аппроксимация:

$$
\begin{aligned}
\alpha_{\mathrm{CRR}} & =1.55 \cdot 10^{-10} T_{e}^{-0.63}+6.0 \cdot 10^{-9} T_{e}^{-2.18}[\mathrm{e}]^{0.37} \\
& +3.8 \cdot 10^{-9} T_{e}^{-4.5}[\mathrm{e}]
\end{aligned}
$$




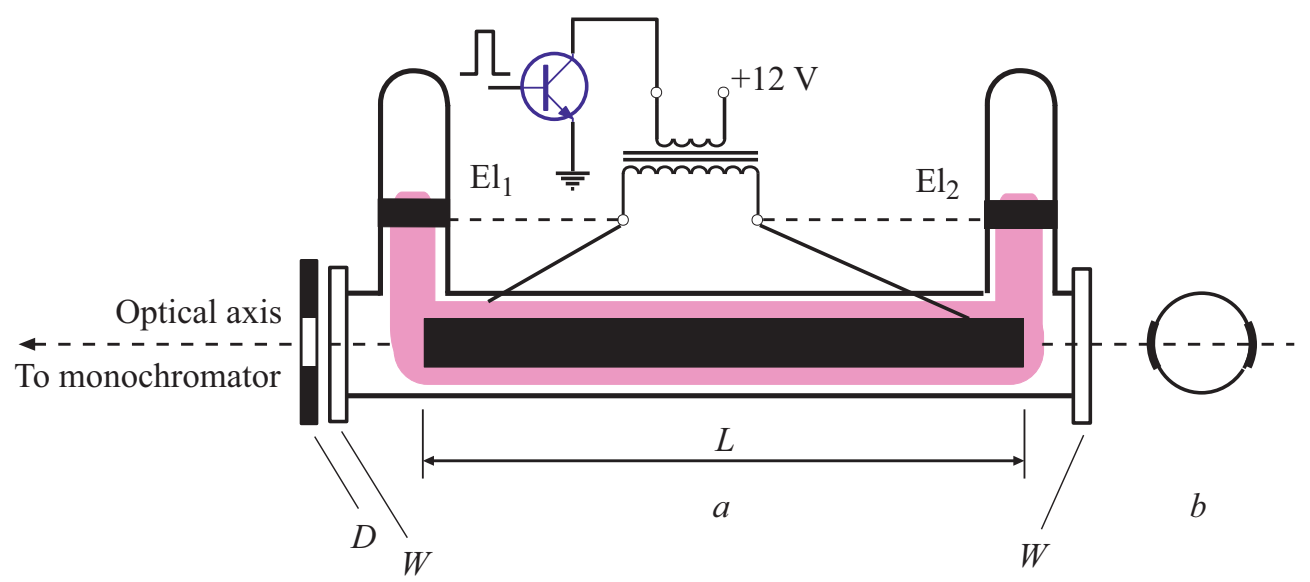

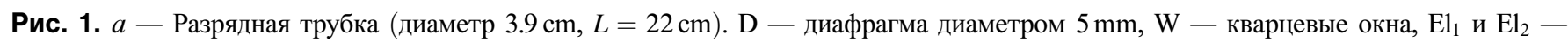
электроды на вертикальных отростках; $b-$ расположение электродов DBD.

Для $[\mathrm{e}]=0.8 \cdot 10^{11} \mathrm{~cm}^{-3}$ и температуры электронов $T_{e}=300 \mathrm{~K} \alpha_{\mathrm{CRR}} \approx 2.5 \cdot 10^{-9} \mathrm{~cm}^{3} / \mathrm{s}$, причем основной вклад в (7) дает третье слагаемое, что указывает на сильную зависимость потока ударно-радиационной рекомбинации от $T_{e}$. С целью учета этого фактора мы внесли второе дополнение к модели [19], состоявшее в учете релаксации температуры электронов в послесвечении на основе данных, представленных в [5].

Специфика DR в гелии требует анализа процессов, формирующих распределение населенностей по колебательным уровням молекулярного иона. Данные о таких процессах, подтвержденные экспериментальными наблюдениями кинетики колебательно-возбужденных ионов $\mathrm{He}_{2}^{+}(v)$ в плазме, отсутствуют. В литературе, однако, доминирует точка зрения, согласно которой скорости колебательной релаксации при столкновениях с частицами плазмы на порядки выше скоростей иных процессов с участием молекулярных ионов. Так, авторы [15] ссылаются на неопубликованную оценку времени жизни молекулярного иона $\mathrm{He}_{2}^{+}(v)$, обусловленного столкновением с атомами гелия: $\tau_{v} \approx(10 \mu \mathrm{s}) / v$ при $P_{\mathrm{He}}=1$ Torr, что для $v=1$ соответствует константе скорости $k_{\text {rel }} \approx 3 \cdot 10^{-12} \mathrm{~cm}^{3} / \mathrm{s}$. Bates [21] предложил еще более высокие скорости релаксации ионов $\mathrm{He}_{2}^{+}(v)$ в гелии, отвечающие константе $k_{\text {rel }} \geq 10^{-10} \mathrm{~cm}^{3} / \mathrm{s}$. Такие скорости исключают наличие в плазме колебательновозбужденных ионов. К аналогичному выводу приводят и оценки [22] скорости релаксации при взаимодействии молекулярных ионов с электронами. В то же время в упомянутых выше экспериментах [12] авторы обнаружили значительное отклонение распределения молекулярных ионов гелия от равновесного, причем ионы в состояниях $\mathrm{He}_{2}^{+}(v=3,4)$ вносили существенный вклад в поток рекомбинации. Заметим, что этой степени возбуждения иона достаточно для появления вследствие DR возбужденных атомов $\mathrm{He}\left(3^{3} D\right)$.

Представленные на рис. 2 модельные расчеты потоков DR (5) выполнены с константами $k_{\text {rel }}(1 \rightarrow 0)=$

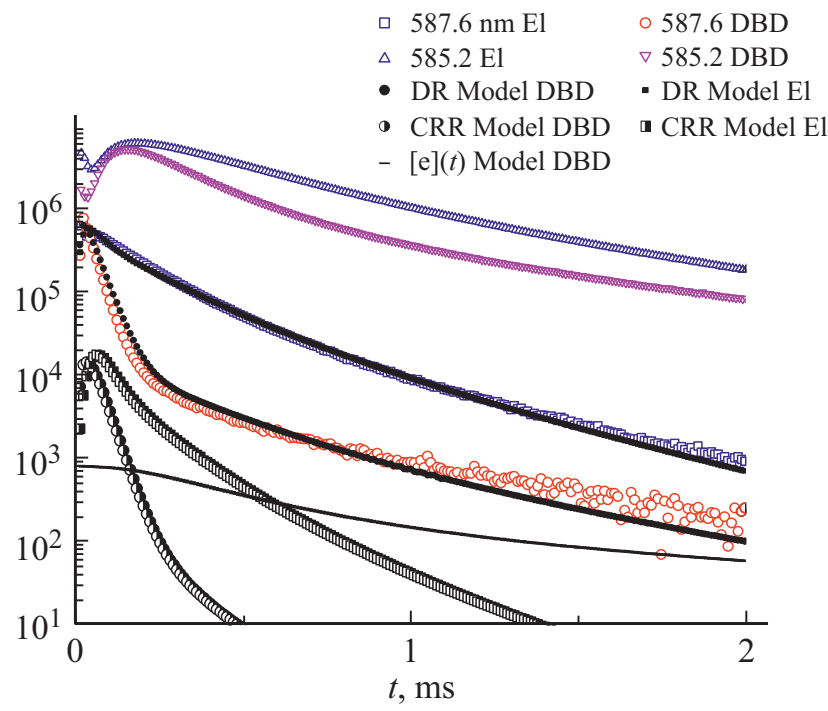

Рис. 2. Интенсивности спектральных линий (в числах фотоэлектронов ФЭУ) гелия и неона в послесвечении двух разрядов и модельные решения (в относительных единицах) для потоков CRR (умножены на 0.25), DR и плотности электронов при $[\mathrm{e}](t=0)=0.8 \cdot 10^{11} \mathrm{~cm}^{-3}$.

$=10^{-12} \mathrm{~cm}^{3} / \mathrm{s} \quad$ и $\quad k_{\mathrm{rel}}(2 \rightarrow 1)=3 \cdot 10^{-12} \mathrm{~cm}^{3} / \mathrm{s} ; \quad$ потоки CRR, вычисленные по (6), (7), умножены на 0.25. В качестве начальных условий для плотностей молекулярных ионов ставились нулевые значения. Важно подчеркнуть, что переход в модели от одного послесвечения к другому осуществлялся изменением только двух параметров - плотностей метастабильных атомов. Видно, что на качественном уровне в рамках модели DR удается достичь значительно более адекватного описания эксперимента. Данные, приведенные на рис. 2, говорят о том, что интенсивности линий гелия $587.6 \mathrm{~nm} \mathrm{и}$ неона $585.2 \mathrm{~nm}$ ведут себя по-разному в ранней фазе послесвечения DBD: интенсивность $587.6 \mathrm{~nm}$ убывает со временем, а интенсивность $585.2 \mathrm{~nm}$ растет. Ход интен- 
сивности линии $587.6 \mathrm{~nm}$ в раннем послесвечении DBD практически совпадает со спадом плотности $\left[\mathrm{He}^{+}\right](t)$ вследствие конверсии (4), что говорит о малой роли процессов с участием метастабильных атомов. То, что интенсивность $J_{587.6}(t) \sim\left[\mathrm{He}^{+}(t)\right]$ на этой стадии распада плазмы достаточно очевидно: при выбранных константах $k_{\text {rel }}$ характерное время $\tau_{\text {rel }}=1 /\left(k_{\text {rel }}[\mathrm{He}]\right)$ релаксации населенностей $\left[\mathrm{He}^{+}(v)\right]$ много меньше времен всех других процессов с участием частиц плазмы, поэтому справедливо квазистационарное решение:

$$
\left[\mathrm{He}_{2}^{+}(v=1)\right] \approx \frac{k_{c}\left[\mathrm{He}^{+}\right][\mathrm{He}]^{2}}{k_{\mathrm{rel}}[\mathrm{He}]}=\left[\mathrm{He}^{+}\right] \frac{\tau_{\mathrm{rel}}}{\tau_{c}}
$$

$\left(\tau_{c}=1 / k_{c}[\mathrm{He}]^{2}-\right.$ время конверсии (4)) притом, что плотность электронов, меняется незначительно. Со временем, как видно из рис. 2, послесвечение DBD приходит к обычно наблюдаемому состоянию, когда в балансе заряженных частиц проявляются и начинают доминировать процессы ионизации типа (1) с участием метастабильных частиц.

Из рис. 2 следует, что поток $F_{\mathrm{DR}}$, вычисленный с указанными выше константами $k_{\text {rel }}$, примерно на порядок превышает $F_{\mathrm{CRR}}$. Как ясно из $(5),(6),(8)$,

$$
\frac{F_{\mathrm{DR}}}{F_{\mathrm{CRR}}}=\frac{\alpha_{\mathrm{DR}}}{\alpha_{\mathrm{CRR}}} \frac{\tau_{\mathrm{rel}}}{\tau_{c}},
$$

что позволяет найти их отношение и при других параметрах $\alpha_{\mathrm{DR}}$ и $\tau_{\text {rel }}$ в рамках квазистационарного подхода. Подчеркнем, что увеличение значений $k_{\mathrm{rel}}$ в модели не меняло характера изменения потоков $F_{\mathrm{DR}}(t)$ и $F_{\mathrm{CRR}}(t)$ со временем, оставляя зависимость $F_{\mathrm{DR}}(t)$ более близкой к $J_{587.6}(t)$ в послесвечении.

Наблюдающийся на рис. 2 рост интенсивности $J_{585.2}(t)$ линии неона $585.2 \mathrm{~nm}$ в начальной фазе послесвечения соответствует увеличению потока DR с электронами ионов $\mathrm{HeNe}^{+}$и $\mathrm{Ne}_{2}^{+}$. Это увеличение потока объясняется возрастанием плотностей $\left[\mathrm{HeNe}^{+}\right](t)$ вследствие последовательности процессов конверсии ионов $\mathrm{He}_{2}^{+}$в $\mathrm{HeNe}^{+}$и $\mathrm{Ne}_{2}^{+}$[23]. Дальнейшее изменение интенсивности $J_{585.2}(t)$ обусловлено зависимостями от времени $[\mathrm{e}](t),\left[\mathrm{HeNe}^{+}\right](t)$ и $\left[\mathrm{Ne}_{2}^{+}\right](t)$.

В заключение отметим, что модели гелиевого послесвечения при давлениях десятки Torr в рамках DR соответствуют и результаты анализа зависимости интенсивностей линий от температуры электронов $J_{\lambda}\left(T_{e}\right)$. Так, наши неоднократные наблюдения отклика $J_{\lambda}(t)$ на импульсный „подогрев“ электронов в послесвечении [24-26] всегда приводили к заключению о типичной для $\mathrm{DR}$ слабой зависимости $J_{587.6}\left(T_{e}\right) \sim T_{e}^{-0.5}$, причем в [23] это получено в эксперименте с одновременным наблюдением неоновых линий, свечение которых было связано с CRR ионов $\mathrm{Ne}^{+}$и следовало температурной зависимости $J_{\lambda}\left(T_{e}\right) \sim T_{e}^{-2}$.

\section{Выводы}

Изучено послесвечение барьерного разряда в гелии с малой добавкой неона, создававшего плазму с малой относительной плотностью метастабильных частиц атомов $\mathrm{He}\left(2^{1} S_{0}\right), \mathrm{He}\left(2^{3} S_{1}\right)$ и молекул $\mathrm{He}_{2}^{*}\left(2 s^{3} \Sigma_{u}^{+}\right)$. Спектроскопические характеристики такой плазмы в течение длительного времени свободны от влияния процессов с участием метастабильных частиц, что позволило, повидимому, впервые в практике эксперимента наблюдать гелиевое послесвечение в условиях чисто рекомбинационной кинетики. Анализ интенсивности $J_{587.6}(t)$ наиболее яркой линии атома гелия $587.6 \mathrm{~nm}$ (переход $\left.\mathrm{He}\left(3^{3} D\right) \rightarrow \mathrm{He}\left(2^{3} P\right)\right)$ проведен в рамках двух моделей: ударно-радиационной рекомбинации ионов $\mathrm{He}^{+}$и диссоциативной рекомбинации колебательно-возбужденных ионов $\mathrm{He}_{2}^{+}$. Сравнение модельных и экспериментальных зависимостей $J_{587.6}(t)$ убеждает в диссоциативном характере заселения состояния $\mathrm{He}\left(3^{3} D\right)$. Таким образом, результаты работы указывают на возможность постановки эксперимента по исследованию механизма диссоциативной рекомбинации молекулярных ионов гелия в наиболее благоприятных для этой задачи условиях.

\section{Конфликт интересов}

Авторы заявляют, что у них нет конфликта интересов.

\section{Список литературы}

[1] Biondi M.A., Brown S.C. // Phys. Rev. 1949. V. 75. N 11. P. 1700. doi 10.1103/PhysRev.75.1700

[2] Florescu-Mitchell A.I., Mitchell J.B.A. // Phys. Rep. 2006. V. 430. P. 277.

[3] Mihajlov A.A., Srećković V.A., Ignjatović Lj.M., Dimitrijević M.S. // MNRAS. 2016. V. 458. P. 2215.

[4] Cŭrík R., Hvizdoš D., Greene C.H. // Phys. Rev. A. 2018. V. 98. P. 062706. doi 10.1103/PhysRevA.98.062706

[5] Deloche R., Monchicourt P., Cheret M., Lambert F. // Phys. Rev. A. 1976. V. 13. N 3. P. 1140. doi 10.1103/PhysRevA.13.1140

[6] Phelps A.V., Brown S.C. // Phys. Rev. 1952. V. 86. N 1. P. 102. doi 10.1103/PhysRev.86.102

[7] Mulliken R.S. // Phys. Rev. 1964. V. 136. N 4A. P. A962. doi 10.1103/PhysRev.136.A962

[8] Bates D.R. // Comments Atom. Mol. Physics. 1976. V. 5. P. 89.

[9] Carata L., Orel A.E., Suzor-Weiner A. // Phys. Rev. A. 1999. V. 59. N 4. P. 2804. doi 10.1103/PhysRevA.59.2804

[10] Johnson R.A., McClure B.T., Holt R.B. // Phys. Rev. 1950. V. 80. N 3. P. 376. doi 10.1103/PhysRev.80.376

[11] Chen C.L., Leiby C.C., Goldstein L. // Phys. Rev. 1961. V. 121. N 5. P. 1391. doi 10.1103/PhysRev.121.1391

[12] Pedersen H.B., Buhr H., Altevogt S., Andrianarijaona V., Kreckel H., Lammich L., de Ruette N., Staicu-Casagrande E.M., Schwalm D., Strasser D., Urbain X., Zajfman D., Wolf A. // Phys. Rev. A. 2005. V. 72. N 1. P. 012712. doi10.1103/PhysRevA.72.012712

[13] Biondi M.A., Holstein T. // Phys. Rev. 1951. V. 82. N 6. P. 962. doi 10.1103/PhysRev.82.962 
[14] Rogers W.A., Biondi M.A. // Phys. Rev. 1964. V. 134. N 5A. P. A1215. doi 10.1103/PhysRev.134.A1215

[15] Ferguson E.E., Fehsenfeld F.C., Schmeltekopf A.L. // Phys. Rev. 1965. V. 138. N 2A. P. A381. doi 10.1103/PhysRev.138.A381

[16] Иванов В.А. // Опт. и спектр. 2019. Т. 126. В. 3. С. 247. doi 10.21883/OS.2019.03.47361.185-18

[17] Phelps A.V. // Phys. Rev. 1955. V. 99. N 4. P. 1307. doi 10.1103/PhysRev.99.1307

[18] Иванов В.А., Приходько А.С., Скобло Ю.Э. // Опт. и спектр. 1991. Т. 70. № 3. С. 507.

[19] Иванов В.А., Петровская А.С., Скобло Ю.Э. // Химическая физика. 2016. Т. 35. № 1. С. 87.

doi 10.7868/S0207401X16010040; Ivanov V.A., Petrovskaya A.S., Skoblo Y.E. // Russ. J. Phys. Chem. B. 2016. V. 10. N 1. C. 153.

[20] Stevefelt J., Boulmer J., Delpech J.-F. // Phys. Rev. A. 1975. V. 12. N 4. P. 1246. doi 10.1103/PhysRevA.12.1246

[21] Bates D.R. // J. Phys. B. 1979. V. 12. N 1. P. L35.

[22] Stevefelt J. // Phys. Rev. A. 1973. V. 8. N 5. P. 2507. doi 10.1103/PhysRevA.8.2507

[23] Veatch G.E., Oskam H.J. // Phys. Rev. A. 1970. V. 2. N 4. P. 1422. doi 10.1103/PhysRevA.2.1422

[24] Иванов В.А., Скобло Ю.Э. // Изв. вузов. Сер. Физика. 1984. T. 2. P. 67; Ivanov V.A., Skoblo Y.E. // Sov. Physics J. 1984. V. 27. N 2. C. $144-148$.

[25] Иванов В.А., Скобло Ю.Э. // ЖЭТФ. 1994. Т. 106. В. 6. C. 1704; Ivanov V.A., Skoblo Yu.E. // J. Exp. Theor. Phys. 1994. V. 79. N 6. P. 921.

[26] Иванов В.А., Петровская А.С., Скобло Ю.Э. // ЖЭТФ. 2019. T. 155. B. 5. C. 901 . doi 10.1134/S0044451019050146; Ivanov V.A., Petrovskaya A.S., Skoblo Y.E. // JETP. 2019. V. $128 . \mathrm{N} 5$. 